\title{
Osteopontin splice variants differentially modulate the migratory activity of hepatocellular carcinoma cell lines
}

\author{
SUJIN CHAE ${ }^{1}$, HYOUNG-OH JUN ${ }^{1}$, EUN GYO LEE ${ }^{2}$, SUK-JIN YANG $^{3}$, DONG CHUL LEE $^{3}$, \\ JOON KI JUNG ${ }^{2}$, KYUNG CHAN PARK ${ }^{3}$, YOUNG IL YEOM ${ }^{3}$ and KYU-WON KIM ${ }^{4}$
}

\author{
${ }^{1}$ NeuroVascular Coordination Research Center and Research Institute of Pharmaceutical Sciences, College of Pharmacy, \\ Seoul National University, Seoul 151-742; ${ }^{2}$ Division of Biotechnology R\&BD, Department of Biomedical \\ Process Development; ${ }^{3}$ Medical Genomics Research Center, KRIBB, Yusong, Daejeon 305-806; \\ ${ }^{4}$ NeuroVascular Coordination Research Center, College of Pharmacy and Department of Molecular \\ Medicine and Biopharmaceutical Sciences, Graduate School of Convergence Science \\ and Technology, Seoul National University, Seoul 151-742, Korea
}

Received April 28, 2009; Accepted June 22, 2009

DOI: 10.3892/ijo_00000458

\begin{abstract}
Osteopontin (OPN, SPP1) is a secretory extracellular matrix protein that has been implicated in cancerassociated mechanisms such as metastasis, invasion and angiogenesis. Three OPN isoforms (OPN-a, -b and -c) derived from alternative splicing are known to exist, but their functional specificity remains unclear. Here, we found that the expression profile of OPN isoforms in hepatocellular carcinoma (HCC) cell lines and patient tissues were correlated with specific cellular phenotypes and tumorigenicity of HCC. Thus, SK-Hep1 cells with a robust migratory capacity dominantly expressed both OPN-a and -b, but non-migratory cell lines such as Hep3B and PLC/PRF/5 mainly expressed OPN-c. Moreover, tumor tissues predominantly expressed OPN-a and -b, whereas normal liver tissues mainly expressed OPN-c. Transwell infiltration and wound-induced migration assays revealed that both OPN-a and -b induced Hep3B cell migration, while OPN-c had no significant effects. By contrast, OPN-c suppressed the migratory activity of SK-Hep1 cells although no significant changes were induced by OPN-a. Consistently, OPN isoforms differentially activated migrationassociated signaling pathways such that OPN-a and -b increased the expression of urokinase type plasminogen activator and the phosphorylation of p42/p44 MAP kinase, but these pathways were not activated by OPN-c. Thus, the findings of the present study suggest that OPN splice variants
\end{abstract}

Correspondence to: Professor Kyu-Won Kim, NeuroVascular Coordination Research Center, College of Pharmacy and Department of Molecular Medicine and Biopharmaceutical Sciences, Graduate School of Convergence Science and Technology, Seoul National University, Seoul 151-742, Korea

E-mail: qwonkim@plaza.snu.ac.kr

Key words: osteopontin, splice variant, migration, hepatocellular carcinoma differentially couple to signaling pathways to modulate the migratory property of HCC cells and that this is one of the mechanisms underlying the pathological heterogeneity of HCC progression.

\section{Introduction}

Osteopontin (OPN) is a secretory extracellular matrix glycoprotein which was first identified in bone tissue as a major sialoprotein to modulate bone formation and remodeling (1). They are expressed in cancer tissues as well, and are involved in various metastasis-associated mechanisms including proliferation, survival, adhesion, migration, invasion and angiogenesis $(2,3)$.

The functional diversity of OPN has been explained at the level of post-translational modification such as phosphorylation, glycosylation, sulfation, enzymatic cleavage and protein crosslinking, causing OPN proteins to differentially bind to seven types of integrins and specific splice variants of CD44 $(2,4)$. For example, the activation of $\alpha v / \beta 3$ integrin receptor by OPN increases the c-Src-mediated phosphorylation of EGFR, activating phosphatidylinositol 3-kinase (PI3K)-dependent Akt and mitogen-activated protein kinase kinase 1 (MEK1)-dependent ERK1/2 pathways (5-7). In contrast, the binding of OPN to CD44 receptors activates the PLC- $\gamma$-dependent Akt pathway $(8,9)$.

Another mechanism underlying the functional diversity of OPN is the existence of splice variants $(a, b, c)$ that have different domain structures: OPN-b lacks the exon 5 from OPN-a, which contains multiple phosphorylation sites, while OPN-c lacks the exon 4 from OPN-a, which contains the transglutaminase domain to induce the polymerization of OPN proteins $(10,11)$. Recently, functional specificity of OPN isoforms has been highlighted; for example, OPN-c is more potent than OPN-a in increasing the anchorage-independence of breast cancer cells (12), and more sensitive to MMP-9dependent cleavage to generate the OPN-5 kDa fragment that increases the invasion of hepatocellular carcinoma (HCC) 
cells (13). However, the functional difference among the three OPN-isoforms has not been extensively studied under the same experimental paradigm.

In the present study, we investigated the effect of three splice variants of OPN proteins on the phenotype of HCC cells and found that different OPN isoforms can differentially modulate the migratory activity of HCC cell lines probably by differentially activating signaling pathways involved in tumor metastasis.

\section{Materials and methods}

Cell culture. HCC cell lines (ATCC, USA) were cultured in Dulbecco's modified Eagle's medium (DMEM) supplemented with $10 \%$ fetal bovine serum (WelGene, Korea) and penicillin/streptomycin (100 units/ml and $100 \mu \mathrm{g} / \mathrm{ml}$ each) (Gibco, Invitrogen, USA), and maintained at $37^{\circ} \mathrm{C}$ in a humidified atmosphere of $5 \% \mathrm{CO}_{2}$. For analysis of secreted proteins, HCC cells were treated with serum-free DMEM for $24 \mathrm{~h}$. HCC cells stably overexpressing OPN were cultured to $80 \%$ confluence before the serum-free medium treatment. HCC cells transiently transfected with OPN expression vectors were grown for an additional $24 \mathrm{~h}$ in fresh $10 \%$ FBS containing DMEM and then changed to serum-free DMEM.

Human tissue samples. Eleven surgically resected HCCs, 4 adjacent non-tumor tissues and 3 normal liver samples obtained from the surgical pathology files of the Catholic University of Korea College of Medicine (CUKCM, Seoul, Korea) were analyzed. All sampling was approved by the Institutional Review Board of CUKCM, and the appropriate consent was obtained.

Plasmids. OPN isoform vectors cloned in pCNS-D2 were obtained from the 21C Frontier Human Gene Bank established by The Center for Functional Analysis of the Human Genome of Korea. Each vector was subcloned into pcDNA ${ }^{\mathrm{TM}} 3.1 /$ $m y c$-His(-) vector. Cloned vectors were confirmed by restriction mapping and sequencing analysis.

Transient transfection. Hep3B cells $\left(2 \times 10^{5}\right)$ were transfected with $1 \mu \mathrm{g}$ OPN plasmid DNA using Lipofectamine Plus Reagent (Invitrogen). After $24 \mathrm{~h}$ of recovery from transient transfection, conditioned media with secreted OPNs were obtained. Cell lysates were analyzed for the cDNA expression level of target genes.

Stable cell lines. Hep3B cells $\left(2 \times 10^{5}\right)$ were transfected with $0.5 \mu \mathrm{g}$ of OPN-encoding plasmid DNA using the Lipofectamine Plus Reagent. Clones expressing each isoform of OPN were selected with G418 (600-1100 $\mu \mathrm{g} / \mathrm{ml})$ for 4 weeks to obtain stable mixed populations.

Western immunoblotting. Cells were harvested in 5X SDS sample buffer [60 mM Tris- $\mathrm{HCl}$ (pH 6.8), 25\% glycerol, $2 \%$ SDS, 14.4 mM 2-mercaptoethanol, 0.1\% bromophenol blue]. Total proteins were resolved in $8-12 \%$ SDS-PAGE gels and specifically detected with anti-OPN (10A16, IBL, Japan), anti-ERK1/2, p-ERK (Cell Signaling Technology, USA) and anti- $\beta$-actin (Sigma, USA) antibodies. The bands were visualized with the ECL Plus System (Amersham Pharmacia Biotech, USA). For secreted proteins, serum-free DMEM was centrifuged at $600 \mathrm{x} \mathrm{g}$ for $5 \mathrm{~min}$ to remove cellular debris, and the supernatant was concentrated 50 -fold by trichloroacetic acid (TCA) precipitation. The precipitated proteins were dissolved with 2X SDS sample buffer and analyzed in SDSPAGE gels.

Reverse transcription and polymerase chain reaction (RT$P C R)$. Total RNA was extracted from HCC cell lines and liver tissues using Trizol reagent (Invitrogen) and RNeasy column (Qiagen GmbH, Germany), respectively, according to the manufacturer's instructions. Total RNA $(5 \mu \mathrm{g})$ was reverse transcribed using MMLV Reverse Transcriptase (Fermentas, Canada). Total OPN transcripts were detected with PCR primer pair: 5'-CCCTTCCAAGTAAGTCCAACGAAAGC-3' (forward) and 5'-CTGGATGTCAGGTCTGCGAAACTTC-3' (reverse). A 30 -cycle PCR was performed at a $58^{\circ} \mathrm{C}$ annealing temperature. Individual OPN isoforms were detected with PCR primer pair: 5'-CATCACCTGTGCCATACCAG-3' (forward) and 5'-GTCAATGGAGTCCTGGCTGT-3' (reverse). A 35-cycle PCR was performed at a $50^{\circ} \mathrm{C}$ annealing temperature. OPN isoform PCR products were separated in $1.2 \%$ agarose gel at a resolution of 20-1000 bp (SeaMatrix; GenoSapiens, Korea). The PCR product was purified from the gel slice using an elution kit (Geneclean ${ }^{\circledR}$ Turbo, MP Biomedicals, LLC). B-actin was amplified by primers: 5'-CA AGAGATGGCCACGGCTGCT-3' (forward) and 5'-TCC TTCTGCATCCTGTCGGCA-3' (reverse). A 19-cycle PCR was performed at a $60^{\circ} \mathrm{C}$ annealing temperature. The urokinase type plasminogen activator (UPA) expression level was determined by primers 5'-TCACCACCAAAATGCT GTGT-3' (forward) and 5'-AGGCCATTCTCTTCCTTGGT-3' (reverse). A 33 -cycle PCR was performed at a $53^{\circ} \mathrm{C}$ annealing temperature. Each of the PCR products representing three splice variants was isolated and confirmed by sequencing analysis.

ELISA assay for OPN secretion. A quantity of OPN isoforms secreted by stable clones was analyzed with the Human Osteopontin Assay Kit (IBL, Japan) according to the manufacturer's instructions. Values at $450 \mathrm{~nm}$ were read, and average counts were calculated.

Colony forming assay. Autoclaved 1\% gum agar (Sigma) was warmed to $55^{\circ} \mathrm{C}$ in a drying oven. Each well of a 6-well plate was filled with $2 \mathrm{ml}$ of $1 \%$ agar in DMEM supplemented with $10 \%$ FBS and solidified at room temperature for $1 \mathrm{~h}$. Hep3B cells $\left(5 \times 10^{4}\right)$ were mixed with $0.7 \%$ soft agar and spread onto the hardened bottom agar. Then cells were cultured at $37^{\circ} \mathrm{C}$ in a humidified $\mathrm{CO}_{2}$ incubator. Culture media were changed every 3 days, and after 3 weeks, colony forming was photographed at x100 or x250 magnification.

Scratch wound migration assay. For the migration assay, a monolayer culture of Hep3B cells grown to $80 \%$ confluence was wounded with a $2 \mathrm{~mm}$-wide yellow tip and then incubated in fresh DMEM supplemented with 10\% FBS. After $65 \mathrm{~h}$, cell migration was photographed at X100 magnification. 
Transwell migration assay. To the outer wells of the transwell insert of a migration plate $(8-\mu \mathrm{m}$ pore size, Corning Costar, \#3422), $10 \mu \mathrm{l}$ of $125 \mu \mathrm{g} / \mathrm{ml} \mathrm{hFN}$ (human fibronectin; BD Biosciences) was added and dried for $1 \mathrm{~h}$ at room temperature. Hep3B cells $\left(5 \times 10^{4} /\right.$ well) stably expressing control or an OPN isoform were prepared by washing once with serum-free DMEM and once with $0.1 \%$ BSA containing DMEM and resuspended in DMEM with $0.1 \%$ FBS. Cells were applied to the inner wells of the coated insert and incubated at $37^{\circ} \mathrm{C}$ in a humidified $5 \% \mathrm{CO}_{2}$ incubator. After 17-24 $\mathrm{h}$, the insert was removed from the well and carefully decanted to remove media from upper wells and wiped out with a clean cotton stick to clean off non-migrated cells. Wells were placed upside down, and the membrane was cut with a razor blade and stained with hematoxylin and eosin (Sigma). The stained well was placed on a glass slide, fixed with xylene and Canada balsam (Sigma) and photographed at x40 and x100 magnification.

Purification of recombinant OPN expressed in $\mathrm{CHO}-\mathrm{KI}$ cells. The OPN-a- or -c-producing cells were established by transfection of a pcDNA3.1(+) vector encoding each of the OPN isoforms into CHO-K1 cells. The protein-free medium (SFM4CHO; Thermo Scientific, Logan, UT) was used for the cell culture. For suspension culture, the $\mathrm{CHO}$ cells were adapted for two weeks. The adaptation was performed by shaking them in 125-ml flasks (Corning Inc., Corning, NY) at $110 \mathrm{rpm}$, in a humidified $5 \% \mathrm{CO}_{2}$ incubator at $37^{\circ} \mathrm{C}$. Batch cultures were conducted in a 2-liter bioreactor (Sartorius BBI, Goettingen, Germany). Exponentially growing cells in a 1-liter spinner flask were inoculated at $2 \times 10^{5}$ cells $/ \mathrm{ml}$ into the bioreactor with a working volume of 2 liters. The agitation speed of the impeller was maintained at $100 \mathrm{rpm}$, and the culture temperature was controlled at $37 \pm 0.1^{\circ} \mathrm{C}$. The dissolved oxygen concentration was controlled at $50 \%$ of air saturation, and the culture $\mathrm{pH}$ was maintained at $7.2 \pm 0.05$ by adding $\mathrm{Na}_{2} \mathrm{CO}_{3}$ or $\mathrm{CO}_{2}$ gas. After four days of culture, the supernatant was collected, centrifuged, and filtered by a microfiltration cartridge $(0.22 \mu \mathrm{m}$, Corning Inc.). The OPN-a- or -ccontaining solution was applied to a Q Sepharose FF column (XK16/20, GE Healthcare, Uppsala, Sweden) equilibrated with $50 \mathrm{mM}$ acetate buffer, $\mathrm{pH}$ 5.8. After washing with $0.2 \mathrm{M}$ $\mathrm{NaCl}$ in equilibrium buffer, OPN proteins were eluted with $0.5 \mathrm{M} \mathrm{NaCl}$ in equilibrium buffer. Eluted proteins were pooled and then dialyzed against distilled water containing diluted phosphate-buffered saline (PBS). Proteins were lyophilized and dissolved in PBS. The concentration of protein was measured using the ELISA Kit (IBL).

Statistical analysis. Statistical comparison between two groups was carried out using the unpaired Student's t-test. Data were expressed as a mean \pm SEM. All data were analyzed by statistical software (Excell, SigmaPlot ${ }^{\mathrm{TM}}$ ).

\section{Results}

Expression profile of OPN splice variants in HCC tissues and cell lines. Osteopontin mRNA is alternatively spliced into three transcripts (10-12). The full form of OPN protein, OPN-a, consists of a part of exon 2 and exons 3, 4, 5, 6 and 7.
Other splice variants, OPN-b and -c, lack the exon 5 (14 amino acids, position 59 to 72 ) or the exon 4 (27 amino acids, position 32 to 58 ), respectively (Fig. 1A).

RT-PCR analysis revealed that OPN splice variants were differentially expressed in HCC cell lines with differential capacity of invasion or migration (14). Thus, SK-Hep1, one type of highly migratory HCC cells, dominantly expressed OPN-a and -b transcripts with barely a detectable level of OPN-c (Fig. 1B). In contrast, non-migratory cell lines, such as Hep3B and PLC/PRF/5 mainly expressed OPN-c. The HepG2 cell line, which was previously reported as nonmigratory (14) but recently has been known to be more adhesive, invasive, and migratory (15), dominantly expressed OPN-a and OPN-b (Fig. 1B).

Migration ability of Hep3B, PLC/PRF/5 and SK-Hep1 cells was confirmed by a transwell migration assay (Fig. 1C), and the order of migratory capacity was found to be SK-Hep1 > PLC/PRF/5 > Hep3B. The Hep3B cells, which was the least migratory and predominantly expressed OPN-c compared to OPN-a and -b, were used in subsequent migration assays.

We additionally analyzed the expression pattern of OPN splice variants in HCC tissues. Normal liver tissues expressed low levels of OPN-a and -b and similar but a slightly higher level of OPN-c (Fig. 1D). In contrast, tumor tissues of HCC dominantly expressed OPN-a and -b but a considerably lower level of OPN-c. The non-transformed tissues isolated from the adjacent region of $\mathrm{HCC}$ exhibited an intermediate expression pattern between the normal liver and HCC tissues as if there was a linear gradient in the expression of different OPN splice variants in association with the progression of HCC (Fig. 1D).

Differential migratory activities of extracellular OPN splice variants. Since the function of OPN proteins is dependent on the proper post-translational modification, recombinant OPN-a and $-\mathrm{c}$ proteins were expressed in and purified from the mammalian system using CHO-K1 cells as previously described (16). The transwell migration assay was performed by extracellularly providing purified recombinant $\mathrm{OPN}-\mathrm{a}$ and OPN-c to SK-Hep1 (Fig. 2A) and Hep3B cells (Fig. 2B). In pro-migratory SK-Hep1 cells, addition of OPN-c inhibited the migratory activities dose-dependently $(\mathrm{p}<0.05)$ (Fig. 2C), while no significant effect was observed by OPN-a treatment (p>0.05) (Fig. 2C). In non-migratory Hep3B cells, OPN-a protein increased cell migration at $10 \mu \mathrm{g} / \mathrm{ml}(\mathrm{p}<0.05)$ (Fig. 2D), but OPN-c treatment showed no significant effects $(\mathrm{p}>0.05)$ (Fig. 2D) .

Pro-migratory activity of $O P N-a$ and $O P N-b$ in Hep $3 B$ cells. Different Hep3B cell lines each overexpressing a distinct OPN isoform were established, and the level of expression was identified by protein blotting (Fig. 3A). To address the causal contribution of OPN isoforms to the migratory nature of HCC cell lines, a scratch wound assay was performed (Fig. 3B). The expression of either OPN-a or -b increased the migration of Hep3B cells by 1.5- to 1.8 -fold ( $p<0.01$ ) (Fig. 3C), while OPN-c expressing cells showed no significant difference when compared with the controls ( $p>0.01)$ (Fig. 3C). Moreover, in transwell migration assays through the 
A

\begin{tabular}{|c|c|c|c|c|c|c|}
\hline \multirow[t]{2}{*}{ OPN-a } & 2 & 3 & 4 & 5 & 6 & 7 \\
\hline & 1 & 18 & & & & \\
\hline OPN-b & 2 & 3 & 4 & & 6 & 7 \\
\hline OPN-c & 2 & 3 & 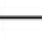 & 5 & 6 & 7 \\
\hline
\end{tabular}

C

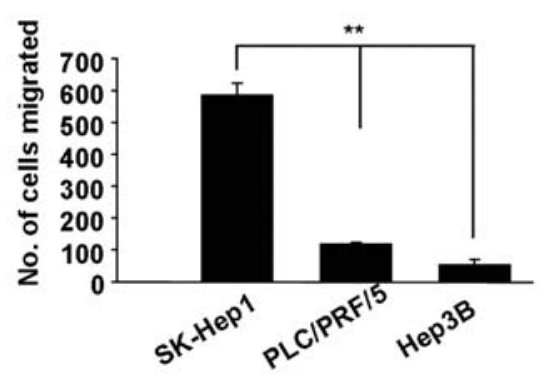

B

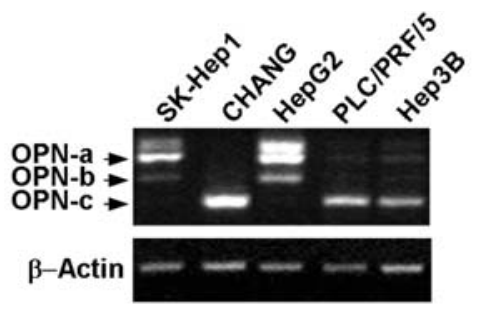

D

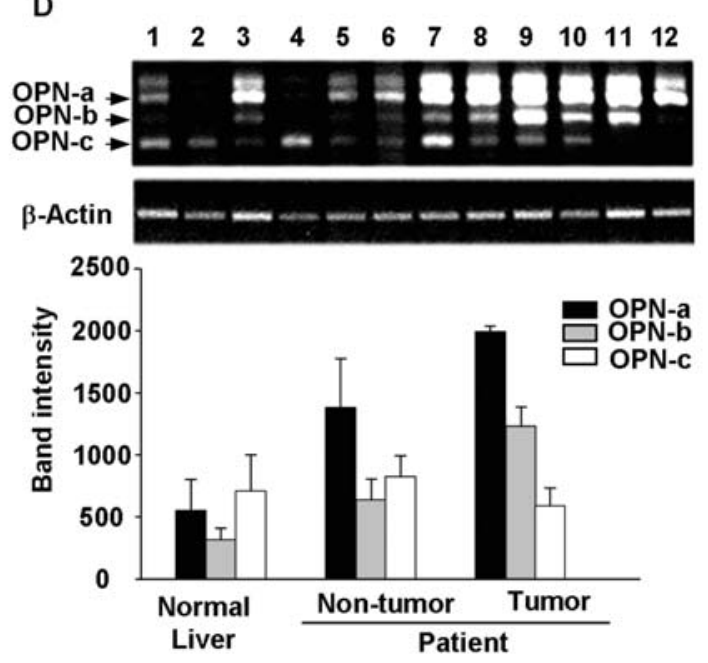

Figure 1. Expression profile of OPN splice variants in hepatocellular carcinoma tissues and cell lines. (A) Exon structure of osteopontin splice variants encoding OPN-a, -b, and -c. Boxes represent exons, and numbers indicate the position of amino acid residues. OPNf and OPNr are forward and reverse primers for PCR reaction, respectively. (B) OPN splice variants identified from cell lines by RT-PCR. HCC cell lines: SK-Hep1, HepG2, PLC/PRF/5 and Hep3B; CHANG (CHANG liver) as an untransformed liver cell line. OPN-a is 277 bp; OPN-b is 235 bp; OPN-c is 195 bp. (C) The number of migrated cells counted after $18 \mathrm{~h}$ in the transwell migration assay. Values are represented as the mean $\pm \mathrm{SEM} .{ }^{* *} \mathrm{p}<0.01$. (D) Expression of OPN splice variants determined from HCC patient tissues by RT-PCR. A representative gel image is shown. Lane 1 and 2, normal tissues; 3-5, surrounding non-transformed liver tissues; 6-12, liver tumor tissues. The amount of PCR product was estimated by densitometry (TINA program version 2.09) (lower panel).

membrane coated with human fibronectin, Hep3B cells stably overexpressing either OPN-a or -b showed significantly higher migratory activities $(\mathrm{p}<0.01)$ (Fig. 3D) than the control but those overexpressing OPN-c showed no significant changes (Fig. 3D) $(\mathrm{p}>0.01)$.

It is of interest to note that OPN-b was more potent in stimulating the migratory activity of Hep3B cells than OPN-a (1.8-fold, p<0.01) (Fig. 3D). To our knowledge, this is the first report on the functional specificity of OPN-b. Consistently, transient expression of OPN isoforms caused similar effects on the migratory activities of Hep3B (OPN-b $>$ OPN-a >> OPN-c, data not shown), comparable to the results from the stable cell lines.

OPN splice variants differentially modulate intracellular signaling pathways in Hep3B cells. To examine the effects of OPN isoforms on the signaling pathways that are involved in cancer cell migration $(8,9)$, conditioned medium collected from OPN-transfected Hep3B cells was tested for its effects on non-transfected control Hep3B cells (Fig. 4).

The transcription of uPA, a key molecule known to regulate cell migration $(8,17)$ and the activation of ERK1/2, which is known to induce uPA in response to integrin signaling was examined (Fig. 4A and B). The uPA level was significantly increased by expression of either OPN-a or $-\mathrm{b}$ $(\mathrm{p}<0.05)$ (Fig. 4C), while it was weakly increased by OPN-c. The phosphorylation of ERK1/2 was significantly increased by OPN-a- or OPN-b-conditioned medium when compared with the control (Fig. 4D) $(\mathrm{p}<0.05)$, but OPN-c-conditioned medium had no significant effects. These results suggest that OPN-a and -b are involved in the activation of integrin signaling as previously described (3) but that OPN-c cannot activate the pathway.

Efficient induction of anchorage-independent growth of Hep3B cells by $O P N-c$. The anti-migratory function of OPN-c first demonstrated in this study seems to be in contrast with previous findings that OPN-c is highly potent in the anchorageindependent growth of breast cancer cells (12). To exclude the effect from the usage of different cell lines, we examined the anchorage-independent growth in Hep3B cells (Fig. 5A). A colony forming assay in soft agar showed that OPN-c was more effective in colony formation of Hep3B cells, as measured by the size of the colonies $(\mathrm{p}<0.01)$ (Fig. 5C), but its effect on the number of colonies was not significantly higher than the others $(\mathrm{p}>0.05)$ (Fig. 5B). These results suggest that $\mathrm{OPN}-\mathrm{c}$ is functional in facilitating the anchorage-independent growth of HCC as previously described in breast cancer (12) and, at the same time, it is less effective than other OPN variants in the promotion of migratory activities. 
A

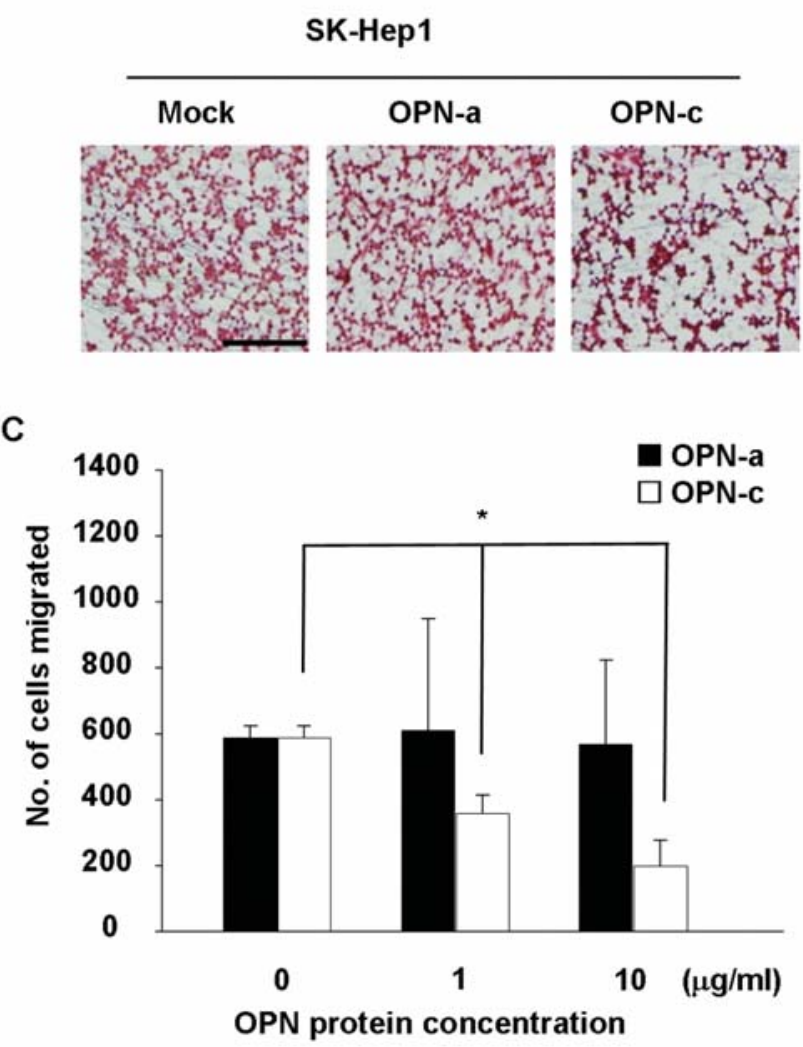

B

Hep3B

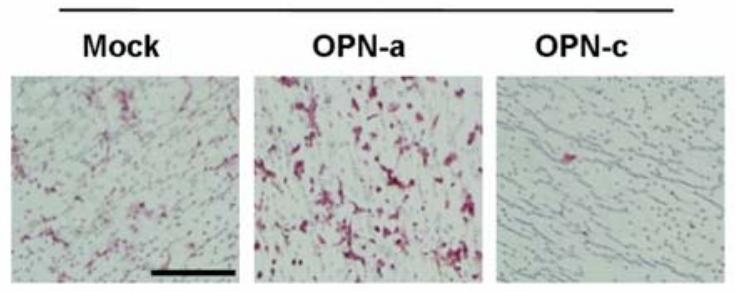

D

OPN-a

$\square$ OPN-c

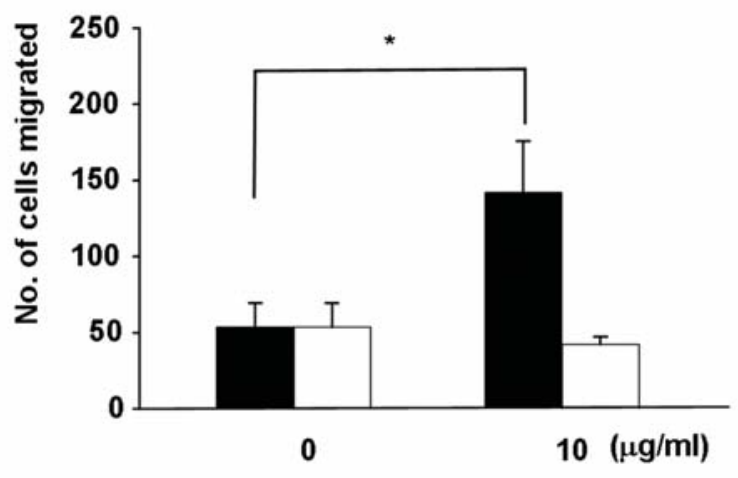

OPN protein concentration

Figure 2. Opposite effects of OPN-a and -c proteins on the migratory capacity of SK-Hep1 and Hep3B cells. (A) The effect of OPN-a and -c proteins on SK-Hep1 cell migration as determined by the transwell migration assay. Note the delay in the migration of OPN-c treated cells. Scale bar, $100 \mu \mathrm{m}$. (B) The effect of OPN-a and -c proteins on Hep3B cell migration as determined by the transwell migration assay. Note the facilitated migration of OPN-a-treated cells. Scale bar, $100 \mu \mathrm{m}$. (C) Quantification of SK-Hep1 cells migrated after treatment with OPN-a or -c in the transwell migration assay. (D) Quantification of Hep3B cells migrated after treatment with OPN-a or $-\mathrm{c}$ in the transwell migration assay. Data are expressed as the mean $\pm \mathrm{SEM}$. "p $<0.05$.

A

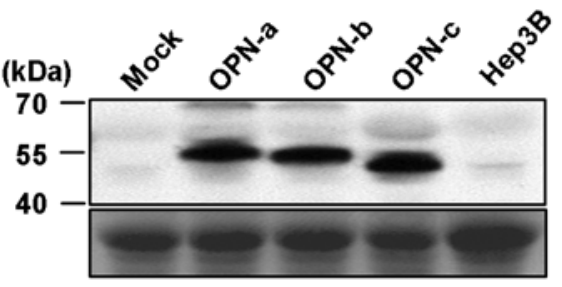

C

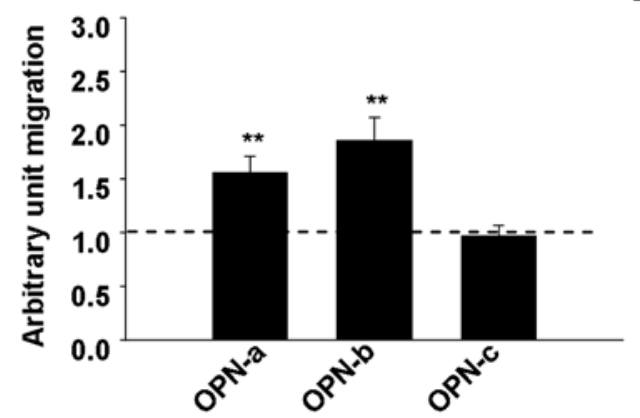

B

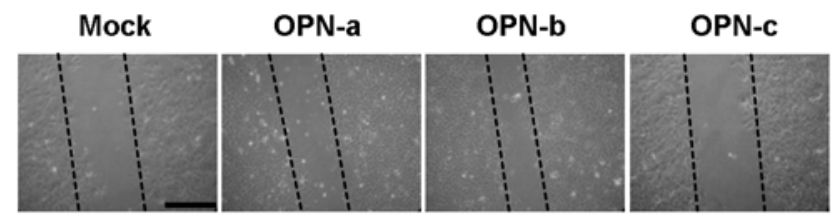

D

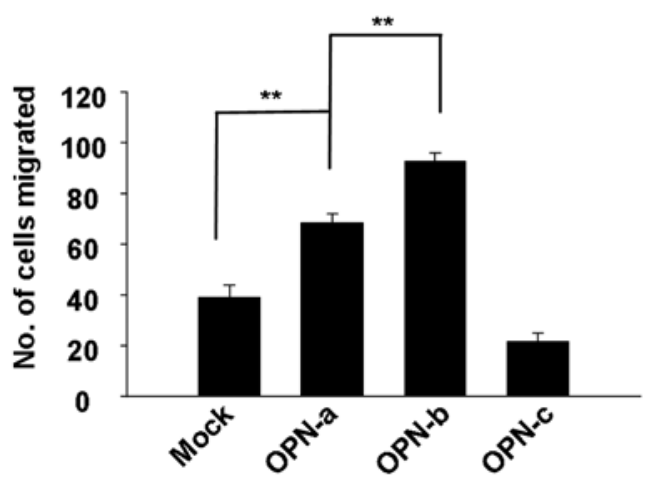

Figure 3. Differential effects of OPN isoforms on the migration of Hep3B cells. (A) Western blotting of OPN proteins secreted from Hep3B cell lines engineered to overexpress OPN splice variants. Numbers indicate the molecular weight of the standard proteins (kDa). Ponceau S stained gel image is shown (lower panel) as a control for proteins in the conditioned media. (B) Scratch wound assay. The wound closure manifested by the migration of Hep3B cells was monitored $65 \mathrm{~h}$ after the wounding. Two dotted lines mark the margin of migrated cells in each slide. Scale bar, $100 \mu \mathrm{m}$. (C) Quantification of the woundbased migration activity as measured by the width of the wound area shortened by cell migration. (D) The migratory capacity measured by the transwell migration assay for $17 \mathrm{~h}$. Values are the mean \pm SEM. ${ }^{* *} \mathrm{p}<0.01$. 
A

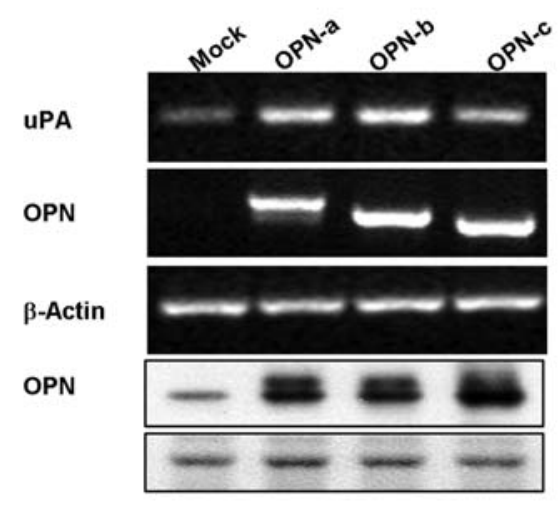

C

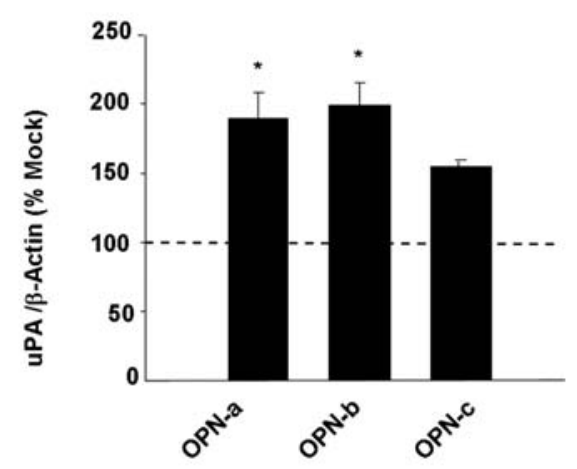

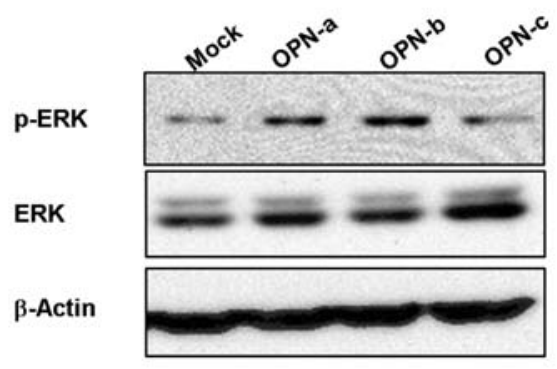

D

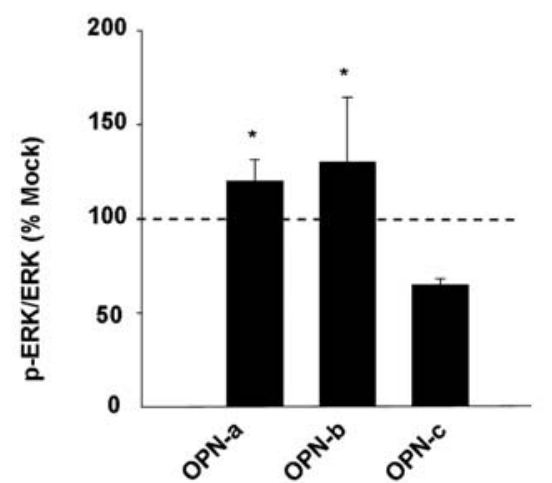

Figure 4. OPN isoforms differentially modulate intracellular signaling pathways in Hep3B cells. (A) Transcripts of uPA were analyzed by RT-PCR in Hep3B cell lines expressing different OPN isoforms. OPN expression was confirmed by both RT-PCR and Western blotting of the conditioned media. The Ponceau S stained gel image indicates the control for secreted proteins in conditioned media (lower panel). (B) Immunoblotting of the activated form (p-ERK) and native form ERK1/2 (p42/p44 MAPK). (C) Quantification of uPA induced by different OPN isoforms. The intensity of the uPA band in A was estimated by densitometry (TINA program version 2.09) using B-actin as the control. (D) Quantification of phospho-ERK1/2 induced by different OPN isoforms. The intensity of phospho-ERK band in B was estimated by densitometry using total ERK as the control. Data in C and D are expressed as the mean \pm SEM. " $\mathrm{p}<0.05$. Dotted line represents the level of the mock.

A
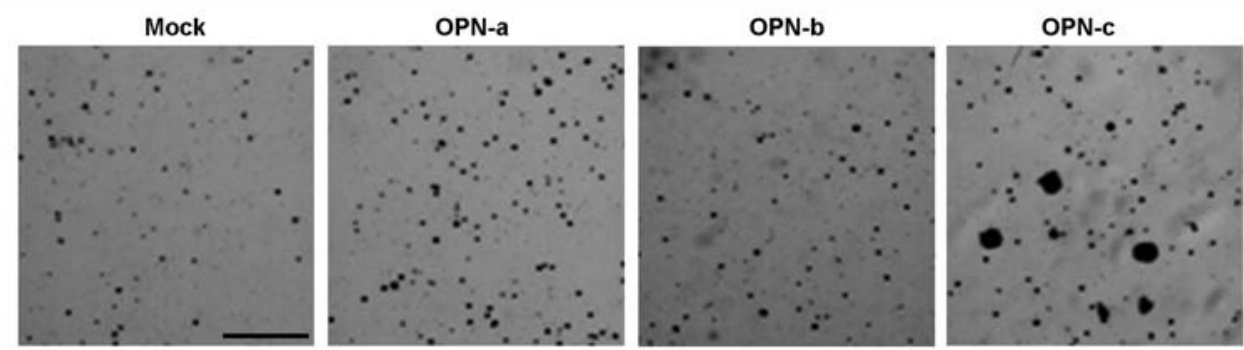

B

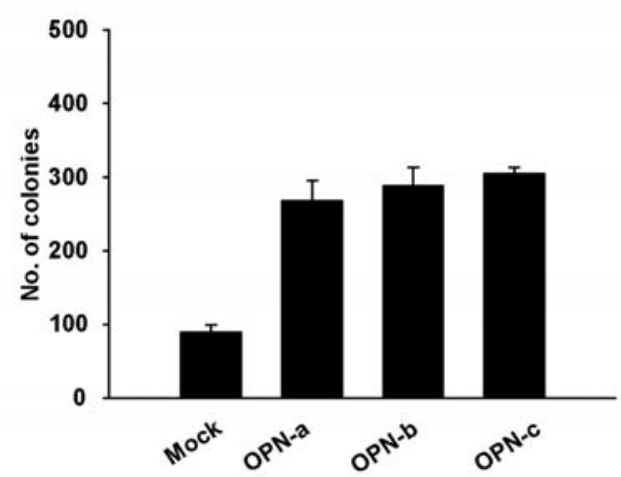

C

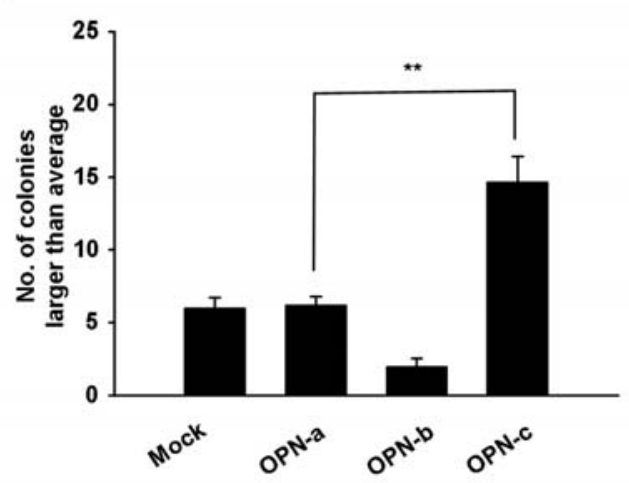

Figure 5. Efficient stimulation of anchorage-independent growth of Hep3B cells by OPN-c. (A) Effect of OPN isoforms on the anchorage-independent growth of Hep3B cells as measured by the colony forming assay. Scale bar, $100 \mu \mathrm{m}$. (B) The average number of colonies counted from four different randomly selected regions of the photographed plate. Values represent the mean \pm SEM. (C) The average number of colonies with diameters $>2 \mathrm{~mm}$. Values are expressed as the mean \pm SEM. ${ }^{* *} \mathrm{p}<0.01$. 


\section{Discussion}

OPN protein has been implicated as a candidate marker for metastatic HCC (18), and it is transcribed into at least three splice variants $(10,11)$. The present study demonstrated the differential expression of OPN splice variants in HCC tissues and cell lines and their possible role in the pathogenesis of HCC.

We used different HCC cell lines with different metastatic capacities to demonstrate the expression and function of OPN isoforms. For example, the SK-Hep1 cell line is highly migratory in a tissue culture condition, while Hep3B and $\mathrm{PLC} / \mathrm{PRF} / 5$ cells are non-migratory in vitro and non-metastatic in vivo (14). As previously described (2) and confirmed in our study, OPN-a and -b isoforms are highly expressed in $\mathrm{HCC}$ tissues. However, OPN-c expression is relatively lower in HCC tissues compared to normal liver (Fig. 1D). In HCC cell lines, SK-Hep1 dominantly expressed OPN-a and -b, while Hep3B and PLC/PRF/5 mainly expressed OPN-c (Fig. 1B). In addition, the differential expression of OPN isoforms could be functional since OPN-c-treated SK-Hep1 cells became less migratory, and the forced expression of either OPN-a or -b made the non-migratory Hep3B cells migratory (Figs. 2 and 3).

Despite the novel anti-migratory role of OPN-c (Fig. 2), this molecule plays a positive role in the anchorage-independent growth of Hep3B cells as well (Fig. 5) as previously described (12). These two findings seem to be inconsistent considering that the anchorage-independent growth and the migratory activity of cancer cells function cooperatively to facilitate the metastasis of cancer $(19,20)$. Cellular adhesion and the migration mechanism, however, can be independent from anchorage-independent growth $(13,21)$. The present results suggest that these two mechanisms can be separated in vitro, and $\mathrm{OPN}-\mathrm{c}$ can be a molecular marker delineating the two processes when the OPN gene plays a role in tumor metastasis $(19,20)$.

Why is OPN-c less effective in facilitating the migration of Hep3B cells? This can be explained by the domain structure of OPN-c which lacks the exon 4 . The exon 4 contains a specialized motif functioning in migration of lymphocytes $(22,23)$. In addition, the exon 4 encodes a transglutaminase domain mediating the polymerization of OPN proteins $(10,11,24)$. The polymeric form of OPN is more effective than the monomer in enhancing cell adhesion, spreading, focal contact formation and migration in SW480 or HUVEC cell lines (25) although the role of polymerization of OPN in HCC cells remains to be studied. Furthermore, a proline-rich region in the exon 4 is known to regulate actin polymerization (26) which may contribute to the migratory activity of HCC cells.

Another unexpected finding is that OPN-b is more effective than OPN-a in supporting the migratory activity of Hep3B cells. Although OPN-b is known to be more vulnerable to proteasome-dependent degradation than OPN-a in breast cancer cells (27), its role in HCC has been largely unknown. Considering the fact that OPN-b lacks the exon 5 that contains two serine phosphorylation sites (4) and is strongly pro-migratory (Fig. 3), the phosphorylation at the exon 5encoded region seems to play a negative role in migration.
Studies involving the missense mutation of the OPN-b protein at these phosphorylation sites in the exon 5 region may elucidate their role in migratory activity.

Multiple domain structures of OPN isoforms could result in differential post-translational modifications, adding to the complexity of OPN binding to target receptors. Supporting this idea, the pro-migratory OPN isoforms (OPN-a or OPN-b) led to the activation of the intergin signaling pathway, as measured by the activation of MAPK and the induction of UPA, to induce cell migration via re-organization of actin cytoskeletons $(8,17,28,29)$, but the anti-migratory OPN-c had no effect on MAPK phosphorylation (Fig. 4).

Based on previous studies and the present findings, the migratory activity of HCC cells highly depends on the expression of different OPN isoforms. Since the migratory activity of cancer cells has been associated with metastasis (30), it is crucial to examine the contribution of different OPN isoforms in the development of HCC in vivo. Analysis of HCC patient tissues indicated that OPN splice variants are differentially expressed during HCC development (Fig. 1D), and that the relative ratio of OPN-a and -b to OPN-c gradually increases as the tumor develops (data not shown). Since OPN-c antagonizes OPN-a and -b in the regulation of cell migration, these results imply that changes in the ratio between levels of OPN-a and -b to that of OPN-c might be an important factor contributing to the progression of HCC. Therefore, it is possible that the expression of OPN-a and -b may reflect the pro-migratory stage of HCC development, while the increase in OPN-c is associated with the nonmigratory and pro-survival stage. How the splicing of OPN is differentially regulated during HCC development also remains to be determined. Further studies on the mechanisms responsible for the alternative splicing and post-translational modification of OPN isoforms will lead to an understanding of the stage-specific typing of HCC.

\section{Acknowledgements}

This work was supported by the Korea Science and Engineering Foundation (KOSEF) grant funded by the Korean government (MEST) through the Creative Research Initiative program (Grant R16-2004-001010010-2008).

\section{References}

1. Prince CW, Oosawa T, Butler WT, et al: Isolation, characterization, and biosynthesis of a phosphorylated glycoprotein from rat bone. J Biol Chem 262: 2900-2907, 1987.

2. Ramaiah SK and Rittling S: Pathophysiological role of osteopontin in hepatic inflammation, toxicity, and cancer. Toxicol Sci 103: 4-13, 2008.

3. Rangaswami H, Bulbule A and Kundu GC: Osteopontin: role in cell signaling and cancer progression. Trends Cell Biol 16: 79-87, 2006.

4. Sodek J, Ganss B and McKee MD: Osteopontin. Crit Rev Oral Biol Med 11: 279-303, 2000.

5. Das R, Mahabeleshwar GH and Kundu GC: Osteopontin induces AP-1-mediated secretion of urokinase-type plasminogen activator through c-Src-dependent epidermal growth factor receptor transactivation in breast cancer cells. J Biol Chem 279: 11051-11064, 2004.

6. Angelucci A, Festuccia C, Gravina GL, et al: Osteopontin enhances the cell proliferation induced by the epidermal growth factor in human prostate cancer cells. Prostate 59: 157-166, 2004. 
7. Tuck AB, Hota C, Wilson SM and Chambers AF: Osteopontininduced migration of human mammary epithelial cells involves activation of EGF receptor and multiple signal transduction pathways. Oncogene 22: 1198-1205, 2003.

8. Das R, Mahabeleshwar GH and Kundu GC: Osteopontin stimulates cell motility and nuclear factor kappaB-mediated secretion of urokinase type plasminogen activator through phosphatidylinositol 3-kinase/Akt signaling pathways in breast cancer cells. J Biol Chem 278: 28593-28606, 2003.

9. Lin YH and Yang-Yen HF: The osteopontin-CD44 survival signal involves activation of the phosphatidylinositol 3kinase/Akt signaling pathway. J Biol Chem 276: 46024-46030, 2001.

10. Young MF, Kerr JM, Termine JD, et al: cDNA cloning, mRNA distribution and heterogeneity, chromosomal location, and RFLP analysis of human osteopontin (OPN). Genomics 7: 491-502, 1990.

11. Saitoh Y, Kuratsu J, Takeshima H, Yamamoto S and Ushio Y: Expression of osteopontin in human glioma. Its correlation with the malignancy. Lab Invest 72: 55-63, 1995.

12. He B, Mirza M and Weber GF: An osteopontin splice variant induces anchorage independence in human breast cancer cells Oncogene 25: 2192-2202, 2006.

13. Takafuji V, Forgues M, Unsworth E, Goldsmith P and Wang XW: An osteopontin fragment is essential for tumor cell invasion in hepatocellular carcinoma. Oncogene 26: 6361-6371, 2007.

14. Giannelli G, Bergamini C, Fransvea E, Marinosci F, Quaranta V and Antonaci S: Human hepatocellular carcinoma (HCC) cells require both alpha3beta1 integrin and matrix metalloproteinases activity for migration and invasion. Lab Invest 81: 613-627, 2001.

15. Emani S, Zhang J, Guo L, Guo H and Kuo PC: RNA stability regulates differential expression of the metastasis protein, osteopontin, in hepatocellular cancer. Surgery 143: 803-812, 2008.

16. Kon S, Maeda M, Segawa T, et al: Antibodies to different peptides in osteopontin reveal complexities in the various secreted forms. J Cell Biochem 77: 487-498, 2000.

17. Busso N, Masur SK, Lazega D, Waxman S and Ossowski L: Induction of cell migration by pro-urokinase binding to its receptor: possible mechanism for signal transduction in human epithelial cells. J Cell Biol 126: 259-270, 1994.

18. Ye QH, Qin LX, Forgues M, et al: Predicting hepatitis B viruspositive metastatic hepatocellular carcinomas using gene expression profiling and supervised machine learning. Nat Med 9: 416-423, 2003.
19. El-Tanani MK, Campbell FC, Kurisetty V, Jin D, McCann M and Rudland PS: The regulation and role of osteopontin in malignant transformation and cancer. Cytokine Growth Factor Rev 17: 463-474, 2006.

20. Weber GF: Molecular mechanisms of metastasis. Cancer Lett 270: 181-190, 2008.

21. Loughran G, Healy NC, Kiely PA, Huigsloot M, Kedersha NL and $\mathrm{O}^{\prime}$ Connor R: Mystique is a new insulin-like growth factor-Iregulated PDZ-LIM domain protein that promotes cell attachment and migration and suppresses anchorage-independent growth. Mol Biol Cell 16: 1811-1822, 2005.

22. Cao Z, Dai J, Fan K, et al: A novel functional motif of osteopontin for human lymphocyte migration and survival. Mol Immunol 45: 3683-3692, 2008.

23. Fan K, Dai J, Wang H, et al: Treatment of collagen-induced arthritis with an anti-osteopontin monoclonal antibody through promotion of apoptosis of both murine and human activated $\mathrm{T}$ cells. Arthritis Rheum 58: 2041-2052, 2008.

24. Sorensen ES, Rasmussen LK, Moller L, Jensen PH, Hojrup P and Petersen TE: Localization of transglutaminase-reactive glutamine residues in bovine osteopontin. Biochem J 304: 13-16, 1994.

25. Higashikawa F, Eboshida A and Yokosaki Y: Enhanced biological activity of polymeric osteopontin. FEBS Lett 581: 2697-2701, 2007.

26. Holt MR and Koffer A: Cell motility: proline-rich proteins promote protrusions. Trends Cell Biol 11: 38-46, 2001.

27. Mirza M, Shaughnessy E, Hurley JK, et al: Osteopontin-c is a selective marker of breast cancer. Int J Cancer 122: 889-897, 2008.

28. Vassalli JD, Sappino AP and Belin D: The plasminogen activator/plasmin system. J Clin Invest 88: 1067-1072, 1991.

29. Tang H, Kerins DM, Hao Q, Inagami T and Vaughan DE: The urokinase-type plasminogen activator receptor mediates tyrosine phosphorylation of focal adhesion proteins and activation of mitogen-activated protein kinase in cultured endothelial cells. J Biol Chem 273: 18268-18272, 1998.

30. Friedl $\mathrm{P}$ and Wolf K: Tumour-cell invasion and migration: diversity and escape mechanisms. Nat Rev Cancer 3: 362-374, 2003. 